\title{
Hot-Jupiters and hot-Neptunes: A common origin?
}

\author{
I. Baraffe ${ }^{1,2}$, G. Chabrier ${ }^{1}$, T. S. Barman ${ }^{3}$, F. Selsis ${ }^{1}$, F. Allard ${ }^{1}$, and P. H. Hauschildt ${ }^{4}$ \\ 1 CRAL (UMR 5574 CNRS), École Normale Supérieure, 69364 Lyon Cedex 07, France \\ e-mail: [ibaraffe; chabrier;fselsis; fallard]@ens-lyon.fr \\ 2 International Space Science Institute, Hallerstr. 6, 3012, Bern, Switzerland \\ 3 Department of Physics and Astronomy, UCLA, Los Angeles, CA 90095, USA \\ e-mail: barman@astro.ucla.edu \\ ${ }^{4}$ Hamburger Sternwarte, Gojenbergsweg 112, 21029 Hamburg, Germany \\ e-mail: yeti@hs.uni-hamburg.de
}

Received 11 March 2005 / Accepted 9 May 2005

\begin{abstract}
We compare evolutionary models for close-in exoplanets coupling irradiation and evaporation due respectively to the thermal and high energy flux of the parent star with observations of recently discovered new transiting planets. The models provide an overall good agreement with observations, although at the very limit of the quoted error bars of OGLE-TR-10, depending on its age. Using the same general theory, we show that the three recently detected hot-Neptune planets (GJ436, $\rho$ Cancri, $\mu$ Ara) may originate from more massive gas giants which have undergone significant evaporation. We thus suggest that hot-Neptunes and hot-Jupiters may share the same origin and evolution history. Our scenario provides testable predictions in terms of the mass-radius relationships of these hot-Neptunes.
\end{abstract}

Key words. planetary systems - stars: individual: GJ436, $\rho$ Cancri, $\mu$ Ara

\section{Introduction}

Determining the evolution and formation mechanism of extrasolar planets in very small orbits, one to two hundred times smaller than Jupiter's orbit, remains a challenging puzzle. This puzzle recently became even more complex when radial velocity surveys of unprecedented accuracy discovered three extrasolar planets similar in mass to Neptune, i.e. 14 to $21 M_{\oplus}$, with orbital periods $P=2.6-9.5$ days and separations $a=$ 0.028-0.09 AU (Santos et al. 2004; McArthur et al. 2004; Butler et al. 2004). An emerging short-period, Neptune-mass, exoplanet population presents us with intriguing questions about their compositions and origins, with potential implications for the understanding of our own solar system. Because of the presence of Jupiter-mass planets at orbital separations even closer than that of these hot-Neptunes, people ruled out the possibility that the latter were born as more massive giant planets which have lost a significant fraction of their envelope. The current belief following this intriguing discovery was then that these hot-Neptunes are of different nature and have different formation and evolutionary histories than their more massive counterparts (Santos et al. 2004; McArthur et al. 2004; Mazeh et al. 2004).

In a recent paper, we explored the effects of evaporation on the evolution of short-period Jovian planets, using a consistent treatment of the interior structure and the irradiated atmospheric structure (Barman et al. 2001; Chabrier et al. 2004; Baraffe et al. 2004). The idea of evaporation is supported by the recent discovery of an extended atmosphere around the transiting exoplanet HD 209458b (Vidal-Madjar et al. 2003, 2004), demonstrating the occurrence of strong atmospheric evaporation and mass loss for short-period irradiated planets. These models could successfully reproduce the observed radius of the transit OGLE-TR-56 (Chabrier et al. 2004) but the case of HD 209458b remains difficult to explain (Baraffe et al. 2003), raising some contention over the general applicability of current evolution theories to hot-Jupiters.

In this Letter, we first show that our models including irradiation effects from the thermal and the high energy fluxes of the parent star are consistent with recent observations of four other new transits, confirming the peculiarity of HD 209458b. Motivated by this success and by the recent discovery of Neptunian exoplanets, we have extended our calculations to the evolution of irradiated and evaporating gaseous planets below the mass of Saturn, down to masses comparable to those of Uranus and Neptune. We show that, contrary to present belief, all of the recent Neptunian objects, along with their shortperiod Jupiter-mass cousins, could have originated from more massive irradiated gas giants which suffered from evaporation induced by the parent star's high-energy radiation. We also present characteristic mass-radius relationships that bear the imprint of irradiation and atmospheric evaporation on a planet's evolution. These predictions will be tested in the very near future by surveys capable of detecting Earth-sized transiting planets. 
Table 1. Properties of transiting extrasolar planets and of their parent star. The following quantities are displayed: the orbital separation, the mass and radius of the planet, the mass, effective temperature, spectral type and radius of the star, the incident flux (see text) and the age when determined.

\begin{tabular}{lccccccccc}
\hline \hline Object & $a(\mathrm{au})$ & $M_{\mathrm{p}}\left(M_{\mathrm{Jup}}\right)$ & $R_{\mathrm{p}}\left(R_{\mathrm{Jup}}\right)$ & $M_{\star}\left(M_{\odot}\right)$ & $T_{\mathrm{eff} \star}(\mathrm{K})$ & $\mathrm{Sp}$. type & $R_{\star}\left(R_{\odot}\right)$ & $\log \left(F_{\mathrm{inc}}\right)$ & Age $(\mathrm{Gyr})$ \\
\hline OGLE-TR-132 $^{a}$ & 0.0306 & $1.19 \pm 0.13$ & $1.13 \pm 0.08$ & 1.35 & 6411 & $\mathrm{~F}$ & 1.43 & 9.35 & $0-1.4$ \\
OGLE-TR-56 $^{b}$ & 0.023 & $1.45 \pm 0.23$ & $1.23 \pm 0.16$ & 1.04 & 6000 & $\mathrm{G}$ & 1.10 & 9.26 & $3 \pm 1$ \\
HD 209458 $^{c}$ & 0.046 & $0.69 \pm 0.02$ & $1.42_{-0.13}^{+0.10}$ & 1.06 & 6000 & $\mathrm{G}$ & 1.18 & 8.72 & $4-7$ \\
OGLE-TR-10 $^{d}$ & 0.042 & $0.57 \pm 0.12$ & $1.24 \pm 0.09$ & 1 & 5800 & $\mathrm{G}$ & 1 & 8.60 & - \\
OGLE-TR-113 $^{e}$ & 0.023 & $1.35 \pm 0.22$ & $1.08_{-0.05}^{+0.07}$ & 0.77 & 4750 & $\mathrm{~K}$ & 0.765 & 8.53 & - \\
TrES-1 $^{f}$ & 0.039 & $0.76 \pm 0.05$ & $1.04_{-0.05}^{+0.08}$ & 0.89 & 5250 & $\mathrm{~K}$ & 0.83 & 8.32 & $2.5 \pm 1.5$ \\
OGLE-TR-11 $^{g}$ & 0.047 & $0.53 \pm 0.11$ & $1.00_{0.06}^{+0.13}$ & 0.82 & 5070 & $\mathrm{G}-\mathrm{K}$ & 0.85 & 8.12 & - \\
\hline
\end{tabular}

${ }^{a}$ Moutou et al. (2005), ${ }^{b}$ Torres et al. (2004), ${ }^{c}$ Cody \& Sasselov (2002), ${ }^{d}$ Konacki et al. (2005), ${ }^{e}$ Bouchy et al. (2004), ${ }^{f}$ Sozzetti et al. (2004), ${ }^{g}$ Pont et al. (2004).

\section{Analysis of newly detected transit planets}

Since the discovery of the two first and well confirmed transits HD 209458b (Charbonneau et al. 2000) and OGLE-TR-56b (Konacki et al. 2003), five new candidates were found very recently, providing more stringent constraints on the theory of irradiated planets. The properties of these new transits and of their parent star are summarized in Table 1, which also provides the incident flux $F_{\text {inc }}$ irradiating the planet, as defined in Baraffe et al. (2003) ${ }^{1}$. Coupling consistently irradiated atmosphere models calculated with the appropriate incident fluxes (Barman et al. 2005) and interior structures, we have calculated the evolution of planets with parameters $\left(a, M_{\mathrm{p}}, F_{\text {inc }}\right)$ characteristic of each of the new observed transits. The evolutionary models and their input physics are described in Baraffe et al. (2003) and references therein. Figure 1 shows a comparison between evolutionary models and observations for all transiting planets. OGLE-TR-10b and HD 209458b are displayed in the same panel, since they have similar properties, in terms of planet mass and incident flux, and the same model is appropriate to describe their evolution. When the age of the system is undetermined, we adopt an arbitrary age ranging from 1 to 5 Gyr.

Besides irradiation, we also take into account the effect of evaporation of the planet gaseous content due to the stellar incident high energy flux, based on the hydrodynamic model of atmospheric evaporation developed recently by Lammer et al. (2003) and applied by Baraffe et al. (2004) to the case of the two first transits HD 209458b and OGLE-TR-56b. The description of the evaporation model and the details of its coupling with the planet evolution can be found in the two aforementioned references. Note that the evaporation rate is based on heating by the age-dependent stellar XUV and Lyman- $\alpha$ radiation, calibrated to observed age-luminosity $L_{\mathrm{XUV}}(t) / L_{\mathrm{bol}}(t)$ relationships (Lammer et al. 2003; Ribas et al. 2005), and takes root on previous studies devoted to escaping atmospheres of the Solar system terrestrial planets (Öpik 1963; Watson et al. 1981). Such hydrodynamic evaporation yields mass loss rates

${ }^{1} F_{\text {inc }}=\frac{1}{2}\left(\frac{R_{\star}}{a}\right)^{2} F_{\star}$, where $R_{\star}$ and $F_{\star}$ are respectively the radius and the total flux of the parent star, and $a$ the orbital separation. in agreement with the observationally determined lower limit

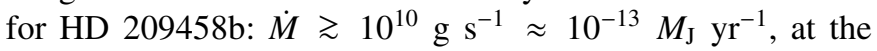
present epoch (Vidal-Madjar et al. 2003).

Models including irradiation and evaporation are shown by the solid lines in Fig. 1, with initial masses of the planets ranging between 0.9 and $2.7 M_{\mathrm{J}}$. These initial conditions remain uncertain, because of the uncertainties in the evaporation model, but the results hold, qualitatively, with different evaporation parameters. Figure 1 shows that consistent irradiated models, including irradiation effects due to both thermal and high energy fluxes of the parent star, are in excellent agreement with the observed radii at the proper ages for all of the planets except HD 209458b and possibly OGLE-TR-10b, depending on its age. Figure 1 also shows that models with evaporation (solid lines) are almost undistinguishable from the case without evaporation (dash-dotted lines). This illustrates the concept of critical mass defined in Baraffe et al. (2004), below which a gaseous planet should evaporate entirely after a certain age, for a given incident flux. The evolution of a planet above this critical mass, however, remains unaffected by evaporation up to several billion years. All the progenitors of the transiting planets fall in this latter domain except, interestingly enough, HD 209458b and OGLE-TR-10b, which are just below this limit. The specific case of HD 209458b was already discussed in Baraffe et al. (2003) and Chabrier et al. (2004). These authors demonstrated, among possible explanations for the particularly large radius, that a mechanism, such e.g. as the one suggested by Guillot \& Showman (2002), which would dissipate a fraction as small as $0.1 \%-0.5 \%$ of the stellar incident flux at the planet internal adiabat level could reproduce the observations of both HD 209458b and OGLE-TR-56b. We have presently tested this hypothesis on OGLE-TR-111, which is the least massive and the least irradiated planet (see Table 1). Adding $0.1 \%$ of the incident stellar energy to the corresponding irradiated model yields a radius larger by $7 \%$ than the upper limit of the observed radius. If a dissipative mechanism, still to be identified, is a common feature of all close-in planets, our test indicates that either it has to be rather inefficient in OGLE-TR-111 or this planet has a significant rocky or icy core, yielding a $\sim 5 \%$ 


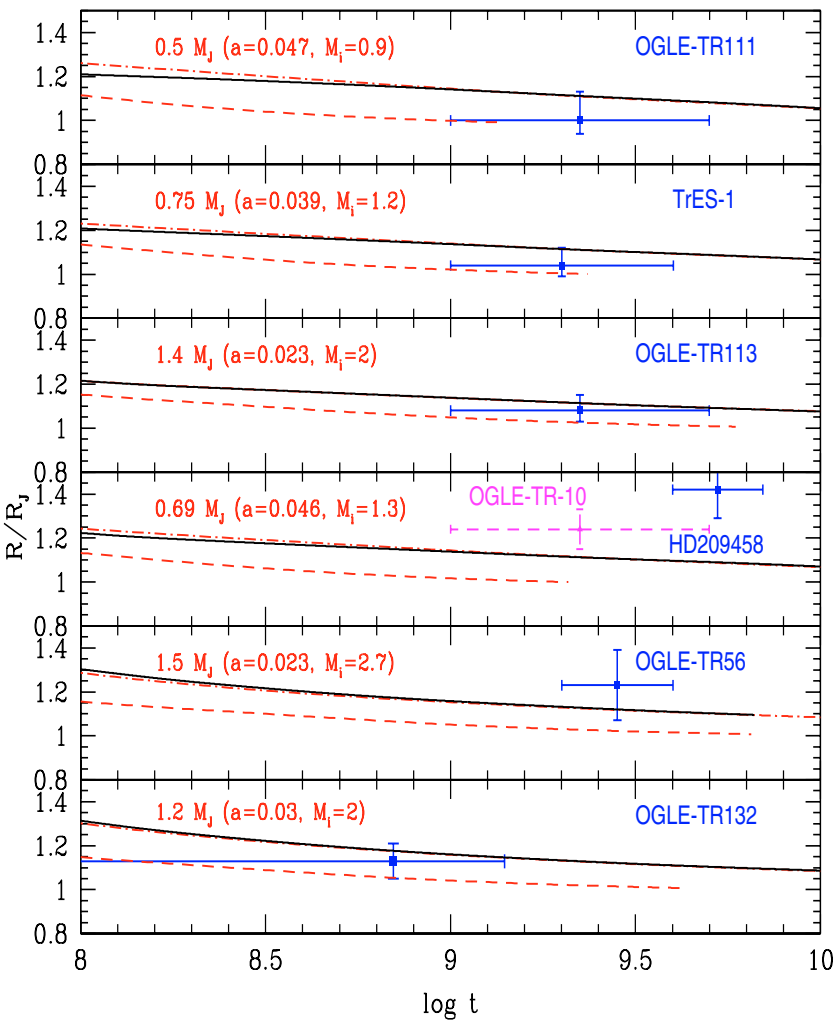

Fig. 1. Evolution of the radius (in $R_{\mathrm{J}}$ ) for currently known transiting planets. The incident stellar flux contribution, $F_{\text {inc }}$, increases from top to bottom. The measured mass and orbital separation are indicated in each panel. The curves correspond to evolutionary models for three cases: without irradiation (dashed lines), with irradiation effects on the thermal structure but no evaporation (dash-dotted lines), and with irradiation and evaporation effects (solid lines). For the latter cases, the initial mass $M_{\mathrm{i}}$ is also indicated in each panel. Note that in most cases the dash-dotted (no evaporation) and solid (evaporation) lines are undistinguishable (see text).

smaller radius than a purely gaseous object (Saumon et al. 1996).

\section{The case of hot-Neptunes}

Current transits do not provide constraints on the evaporation rates, since, in their mass range, their mass-radius relationship remains unaffected by the process of evaporation. However, the good agreement of present models with most of the currently observed transits gives us confidence on a scenario based on a global process of irradiation and evaporation. We have thus extended this scenario to initial masses below the critical mass in order to explore the regime of sub-Jovian mass planets.

Figure 2 displays the evolution of hydrogen/helium planets with initial masses ranging from 0.5 to $1 M_{\mathrm{J}}$ orbiting a parent star under conditions characteristic of the three newly discovered Neptunian planets, namely 0.028 AU from an M 2.5 star (GJ436, Butler et al. 2004), 0.038 AU from a G8 star ( $\rho^{1}$ Cancri, McArthur et al. 2004) and 0.09 AU from a G5 star ( $\mu$ Ara, Santos et al. 2004). The observed spectra and magnitudes of the parent stars, complemented by well established age-activity relations for similar type stars, imply that these systems must be

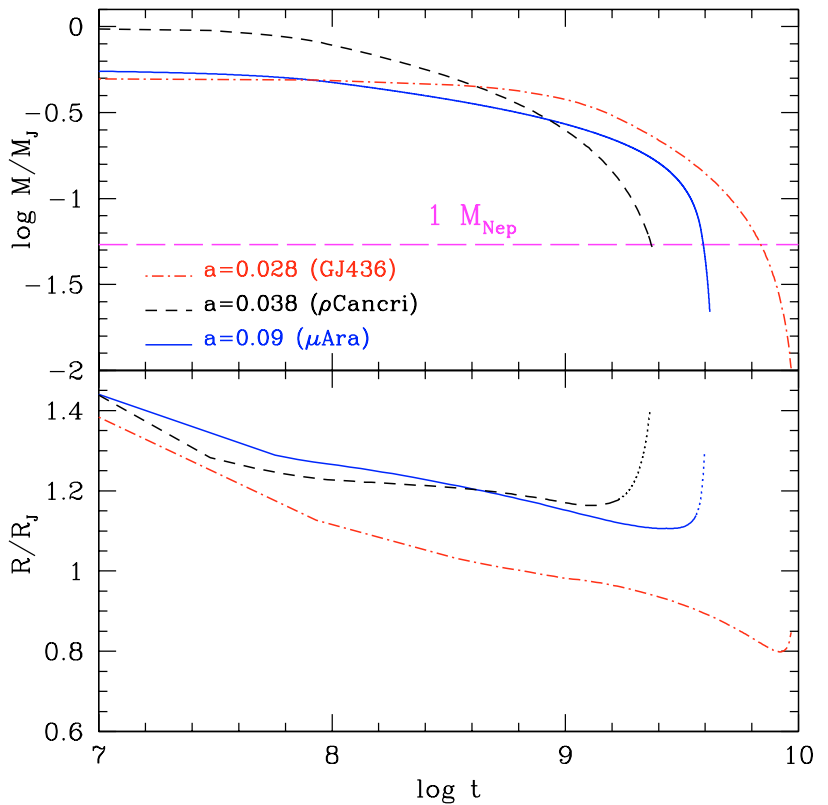

Fig. 2. Evolution of evaporating giant planets reaching a Neptunemass (indicated in the top panel by the long-dashed line, in Jupitermass units $M_{\mathrm{J}}$ ) within a few Gyr. Three planet-star cases are shown for: the G5 star $\mu$ Ara (Santos et al. 2004) (solid), the G8 star $\rho^{1}$ Cancri (McArthur et al. 2004) (dashed), and the M 2.5 star GJ 436 (Butler et al. 2004) (dash-dotted). The planet's orbital separation $a$ (in AU) for each system is indicated in the upper panel.

a few billion year old. After $\sim 2-5$ Gyr, these planets have lost more than $90 \%$ of their initial mass, about 0.5 to 0.95 Jupiter mass of gas, and ultimately become Neptune-mass planets. Note that this evaporation mechanism holds whether or not the planet has migrated inward from larger orbits since its formation. Migration implies the presence of a disk and upper limits for disk lifetimes are $\sim 10 \mathrm{Myr}$ (Armitage et al. 2003). Thus, regardless of migration, the planet will spend the majority of its life in its final short-period orbit, evolving under the influence of irradiation and evaporation.

Given the large amount of mass lost by the planet, one may wonder about the consequences on the planetary orbit. A tentative answer can be inferred from an analogy with the orbital evolution of comets. If matter escapes isotropically, the planetary orbit remains unaffected. If mass loss occurs anisotropically, however, the orbit can be affected. Like for comets, mass loss will take place on the irradiated side of the planet and thus will occur initially in the direction of the parent star, exerting a force in the opposite direction and pushing the planet away from the star. This may affect the evaporation process and eventually quench it, if the orbital separation $a$ increases sufficiently. A quantitative estimate of this effect will depend on the fraction of anisotropy of the escaping flow and its velocity. Such a study is beyond the scope of the present paper. However, the existence of close-in planets on very short orbits $(a<0.05 \mathrm{AU})$ suggests that this effect is small. This question definitly deserves a more thorough analysis.

Note that in our model the upper atmosphere, where most of the incident XUV flux is absorbed, always remains within the Roche lobe radius of the planet. Above this limit, $\sim 1.3 R_{\mathrm{J}}$ 
for $\rho^{1}$ Cancri and $\mu$ Ara, Roche lobe overflow will occur, leading also to complete escape of the planet gas envelope ( $\mathrm{Gu}$ et al. 2003; Lecavelier et al. 2004). The runaway of the evaporation process (Baraffe et al. 2004) leads to the catastrophic expansion of the planets, as indicated by the dotted lines in the bottom panel, and eventually to the evaporation of the entire hydrogen/helium content. The mass-radius relationships predicted by our model are totally different from the one characteristic of a non-irradiated, non-evaporating, $\mathrm{H} / \mathrm{He}$ Neptune-mass planet which, to first approximation, would have a radius $R \sim 0.6 R_{\mathrm{J}}$ after 1 Gyr (Zapolsky \& Salpeter 1969).

\section{Discussion and conclusion}

Our evolutionary models, accounting for both irradiation and evaporation processes, show that most of the close-in exoplanets discovered today, including the emerging Neptune-mass population, may originate from significantly more massive Jupiter-like gas giants which have experienced drastic mass loss due to the stellar high-energy radiation. This result is in direct opposition to the current belief that the hot-Neptunes are of a different nature than the hot-Jupiters. This statement was used in a recent paper by Mazeh et al. (2004) who report an interesting anti-correlation between mass and orbital period of transiting planets. In their analysis, they ignored the recently discovered hot-Neptunes because of their supposedly different nature. We show here that this supposition is by no means obvious and that Jupiter-mass and Neptune-mass close-in planets may share the same origin. Including the Neptune-mass planets in the sample of all short period planets weakens the case for the anti-correlation of Mazeh et al. (2004).

Our scenario is certainly affected by the large uncertainties in the evaporation model of Lammer et al. (2003), which is based on the assumption that the planet undergoes maximal energy-limited evaporation. This idea of energy-limited evaporation, meaning that the escape rate is essentially determined by the amount of the high energy flux absorbed in the upper planetary atmosphere, was originally suggested by Öpik (1963), and further applied by Watson et al. (1981) and many others (see references in Lammer et al. 2003) to terrestrial planet atmospheres. As recently shown by Yelle (2004), energy-limited escape rates for close-in giant planets may be limited by efficient cooling due to chemical species, such as $\mathrm{H}_{3}^{+}$ and $\mathrm{H}^{+}$, which decreases the efficiency of local heating due to the absorption of the XUV flux by the upper atmosphere. The absence of detailed chemistry in the work by Lammer et al. (2003) may result in overestimated rates, as suggested by Yelle (2004). Indeed, this latter finds escape rates 20 times smaller than the ones estimated by Lammer et al. (2003). With such significantly smaller rates, hot-neptunes can not originate from Jupiter- or even Saturn-mass planets. Test calculations with an escape rate 10 times smaller than the one predicted by Lammer et al. (2003) indicate that initial masses less than $\sim 0.1 M_{\text {J }}$ are required to reach a Neptune-mass with the orbital parameters of the three discovered hot-Neptune systems within a few Gyr. The formation of such low mass gaseous planets at such small orbital distances seems to be very unlikely within the framework of current formation models of giant planets.
This certainly would cast doubt on our present suggestion of a common origin for close-in Neptunian and Jovian planets.

The work by Lammer et al. (2003) and Yelle (2004) are the first attempts to understand all the complex processes involved in the evaporation of close-in giant planets. It is presently impossible to favor one of these models over the other. Since the Lammer et al. (2003) model yields maximal energy-limited escape, it is important to examine its consequences on the planet fate as an upper limit case for the evaporation. The next step is to explore systematically the effect of lower evaporation rates, as well as of other uncertainties inherent to our calculations, mainly the effect of initial conditions, of a rocky/icy core and of non-standard chemical composition in the envelope. This work is under progress.

The discovery of Neptunian-mass transiting planets will be crucial for testing the present scenario. As shown in Sect. 3, the proposed irradiation and evaporation global process will be directly revealed by the planet mass-radius relationship. As mentioned previously, a purely gaseous $(\mathrm{H} / \mathrm{He})$ Neptunemass planet not affected by irradiation and evaporation during its evolution will have a radius $R \sim 0.6 R_{\mathrm{J}}$ at present epoch ( $>1$ Gyr). Planets composed dominantly of solid material, like terrestrial planets or the ice giants, Neptune and Uranus, will have significantly more compact structures (Neptune's radius is $\sim 0.35 R_{\mathrm{J}}$ at its present age). In contrast, if irradiation plus evaporation is a dominant process in the evolution of shortperiod exoplanets, with Neptune-mass planets or even smaller objects originating from Jupiter-like gas giants, we predict significantly larger radii for a given mass, namely $R \gtrsim 0.8 R_{\mathrm{J}}$ for the presently detected Neptune-mass objects, as illustrated in Fig. 2.

The large radii we predict for Neptune mass planets place them well within the detection limit of most transit surveys and the discovery of such an object would strongly support our scenario. However, given the low probability of transit detections, no Neptune-mass transit discovery remains consistent with the low number of transits detected up to now. If no object with the presently predicted mass-radius relationships is found, once larger transit statistics become available, then several possibilities exist. Perhaps the evaporation mechanism is not a dominant process in planet history, questioning the observed evaporation of HD 209458b and/or the validity of the present evaporation rates. Or, conceivably, all the progenitors of Neptune-mass objects lie below the predicted critical mass (see bottom panel of Fig. 2) and, at most, a rocky core remains after evaporation. This latter case would suggest that many of the short-period low-mass planets should be made mostly of rocks.

On the other hand, transit detections of Neptune-mass objects with Jupiter-like radii would superbly corroborate the present scenario of irradiated and evaporating gaseous giants. Our ability to find transiting planets will improve significantly with the soon to be launched COROT and KEPLER missions. Comparisons between these observations and the present calculations will potentially bring information about the composition, evolution, and the origin of short-period exoplanets, unveiling part of their mystery and possibly changing the understanding of our own Solar system. 
Acknowledgements. I.B. thanks ISSI (Bern) for hospitality during elaboration of part of this work.

\section{References}

Armitage, P. J., Clarke, C. J., \& Palla, F. 2003, MNRAS, 342, 1139 Baraffe, I., Chabrier, G., Barman, T., Allard, F., \& Hauschildt, P. H. 2003, A\&A, 402, 701

Baraffe, I., Selsis, F., Chabrier, G., et al. 2004, A\&A, 419, L13

Barman, T., Hauschildt, P. H., \& Allard, F. 2001, ApJ, 556, 885

Barman, T., Allard, F., \& Hauschildt, P. H. 2005, in preparation

Bouchy, F., Pont, F., Santos, N. C., et al. 2004, A\&A, 421, L13

Butler, R. P., Vogt, S. S., Marcy, G. W., et al. 2004, ApJ, 617, 580

Chabrier, G., Barman, T., Baraffe, I., Allard, F., \& Hauschildt, P. 2004, ApJ, 603, L53

Charbonneau, D., Brown, T., Latham, D., \& Mayor, M. 2000, ApJ, 529, L45

Cody, A. M., \& Sasselov, D. D. 2002, ApJ, 569, 451

Guillot, T., \& Showman, A. P. 2002, A\&A, 385, 156

Gu, P., Lin, D. N. C., \& Bodenheimer, P. H. 2003, ApJ, 588, 509

Konacki, M., Torres, G., Jha, S., \& Sasselov, D. 2003, Nature, 421, 507
Konacki, M., Torres, G. S., Sasselov, D., \& Jha, S. 2005, ApJ, in press [arXiv: astro-ph/0412400]

Lammer, H., Selsis, F., Ribas, I., et al. 2003, ApJ, 598, L121

Lecavelier des Etangs, A., Vidal-Madjar, A., McConnell, J. C., \& Hébrard, G. 2004, A\&A, 418, L1

Mazeh, T., Zucker, S., \& Pont, F. 2004, MNRAS, 356, 955

McArthur, B. E., Endl, M., Cochran, W. D., et al. 2004, ApJ, 614, L81

Moutou, C., Pont, F., Bouchy, F., \& Mayor, M. 2004, A\&A, 424, L31

Öpik, E. J. 1963, Geophys. J. RAS, 7, 490

Pont, F., Bouchy, F., Queloz, D., et al. 2004, A\&A, 426, L15

Ribas, I., Guinan, E. F., Güdel, M., \& Audard, M. 2005, ApJ, 622, 680

Santos, N. C., Bouchy, F., Mayor, M., et al. 2004, A\&A, 426, L19

Saumon, D., Hubbard, W. B., Burrows, A., et al. 1996, ApJ, 460, 993

Sozzetti, A., Yong, D., Torres, G., et al. 2004, ApJ, 616, L167

Torres, G., Konacki, M., Sasselov, D., \& Jha, S. 2004, ApJ, 609, 1071

Vidal-Madjar, A., Lecavalier des Etangs, A., Désert, J.-M., et al. 2003, Nature, 422, 143

Vidal-Madjar, A., Désert, J.-M., Lecavalier des Etangs, A., et al. 2004, ApJ, 604, L69

Watson, A. J., Donahue, T. M., \& Walker, J. C. G. 1981, Icarus, 48, 150

Yelle, R. V. 2004, Icarus, 170, 167

Zapolsky, H. S., \& Salpeter, E. E. 1969, ApJ, 158, 809 